\title{
BULLETIN DES SCIENCES
}

\section{MATHÉMATIQUES ET ASTRONOMIQUES}

\section{HERMITE}

\section{Sur la réduction des intégrales hyperelliptiques aux fonctions de première, de seconde et de troisième espèce}

Bulletin des sciences mathématiques et astronomiques $2^{e}$ série, tome 7, no 1 (1883), p. 36-42

<http://www.numdam.org/item?id=BSMA_1883_2_7_1_36_1>

(C) Gauthier-Villars, 1883, tous droits réservés.

L'accès aux archives de la revue «Bulletin des sciences mathématiques et astronomiques » implique l'accord avec les conditions générales d'utilisation (http://www.numdam.org/conditions). Toute utilisation commerciale ou impression systématique est constitutive d'une infraction pénale. Toute copie ou impression de ce fichier doit contenir la présente mention de copyright.

\section{NumDam}

Article numérisé dans le cadre du programme

Numérisation de documents anciens mathématiques

http://www.numdam.org/ 


\section{MÉ L A N GES.}

\section{SUR LA RÉDUGTION DES INTÉGRALES HYPERELLIPTIQUES AUX FONGTIONS DE PREMIËRE, DE SECONDE ET DE TROISILMEE ESPĖGE;}

Pir M. HER IITE. .

L'étude des intégrales hyperelliptiques de la forme $\int \frac{\mathrm{P} d x}{\mathrm{Q} \sqrt{\mathrm{R}}}$, où $\mathrm{P}, \mathrm{Q}$ et $\mathrm{R}$ sont des polynômes quelconques en $x$, dont le dernier est supposé n'avoir point de facteurs multiples, s'ouvre par la réduction à un terme algébrique et aux fonctions de première, de seconde et de troisième espèce. La méthode consiste à décomposer, en une parlie entière et en fractions simples, la fraction rationnelle $\frac{\mathrm{P}}{\mathrm{Q}}$, ce qui ramène d'abord aux quantités $\int \frac{x^{m} d x}{\sqrt{\mathrm{R}}}$, 
$\int \frac{d x}{(x-\alpha)^{p+1} \sqrt{\mathbf{R}}}$; on fait voir ensuite que, en désignant le degré de $\mathrm{R}$ par $r$, la première peut être réduite pour toute valeur de $m$, aux cas où cet exposant ne surpasse pas $r-2$, tandis que la seconde se ramène, par un procédé analogue, au seul cas de $p=0$.

Dans une leçon à la Sorbonne, je me suis placé à un point de vue différent pour traiter cette question importante, et je vais l'indiquer en peu de mots.

Je partirai de la forme du dénominateur $Q$, que donne la méthode des racines égales, à savoir

$$
\mathrm{Q}=\mathrm{A}^{\mathrm{x}+1} \mathrm{~B}^{\mathrm{s}+1} \mathrm{C}^{\gamma+1} \ldots,
$$

A, B, C, ... n'ayant que des facteurs simples; mais, en outre, je mettrai à part, s'il y a lieu, ceux de ces facteurs qui appartiennent au polynòme R. Dans ce cas, j'adopterai l'expression suivante :

$$
\mathrm{Q}=\mathrm{A}^{\alpha+1} \mathrm{~B}^{\beta+1} \mathrm{C}_{i+1} \ldots \mathrm{S}^{\sigma} \mathrm{T}^{\tau} \ldots,
$$

où $\mathrm{S}, \mathrm{T}, \ldots$ sont des diviseurs de $\mathrm{R}$, et il me sera ainsi permis de supposer A, B, C, ... premiers avec $R$.

Cela étant, nous pouvons écrire, en désignant par $\mathbf{G}, \mathbf{H}, \ldots, \mathbf{M}$ dès polynômes entiers

$$
\frac{\mathrm{P}}{\mathrm{Q}}=\frac{\mathrm{G}}{\mathrm{A}^{\alpha+1}}+\frac{\mathrm{H}}{\mathrm{B}^{\beta+1}}-\cdots+\frac{\mathrm{M}}{\mathrm{S}^{\sigma}}+\cdots,
$$

et cette expression conduit à deux types d'intégrales

$$
\int \frac{\mathrm{G} d x}{\mathrm{~A}^{\alpha+1} \sqrt{\bar{R}}} \text { et } \int \frac{\mathrm{M} d x}{\mathrm{~S}^{\sigma} \sqrt{\mathrm{R}}}
$$

que je vais considérer successivement.

Je ferai d'abord dans la première

$$
\mathrm{G}=\mathbf{A X}+\mathbf{A}^{\prime} \mathbf{R Y},
$$

en remarquant qu'on satisfait à cette condition, au moyen de polynômes entiers pour $\mathrm{X}$ et $\mathrm{Y}$, attendu que $\mathrm{A}$ et $\mathrm{A}^{\prime} \mathrm{R}$ n'ont aucun facteur commun, d'après ce que nous avons supposé. Nous aurons donc

$$
\int \frac{\mathrm{G} d x}{\mathrm{~A}^{\alpha+1} \sqrt{\overline{\mathrm{R}}}}=\int \frac{\mathrm{X} d x}{\mathrm{I}^{x} \sqrt{\mathrm{R}}}+\int \frac{\mathrm{A}^{\prime} \mathrm{Y} \sqrt{\mathrm{R}}}{\mathrm{A}^{\alpha+1}} d x
$$


38

PREMIÈRE PAR'IIE.

ou encore

$$
\int \frac{\mathrm{G} d x}{\mathrm{~A}^{\alpha+1} \sqrt{\mathrm{R}}}=\int \frac{\mathrm{X} d x}{\mathrm{~A}^{\alpha} \sqrt{\mathrm{R}}}-\frac{\mathrm{I}}{\alpha} \int \mathrm{D}_{x}\left(\frac{\mathrm{I}}{\mathrm{A}^{\alpha}}\right) \mathrm{Y} \sqrt{\mathrm{R}} d x .
$$

Mais on obtient, en intégrant par parties,

$$
\int \mathrm{D}_{x}\left(\frac{\mathrm{I}}{\mathrm{A}^{\alpha}}\right) \mathrm{Y} \sqrt{\mathrm{R}} d x=\frac{\mathrm{Y} \sqrt{\mathrm{R}}}{\mathrm{A}^{\alpha}}-\int \frac{\mathrm{D}_{x}(\mathrm{Y} \sqrt{\mathrm{R}})}{\mathrm{A}^{\alpha}} d x,
$$

et l'on en conclut la relation

$$
\int \frac{\mathrm{G} d x}{\mathrm{~A}^{\alpha+1} \sqrt{\mathbf{R}}}=\int \frac{\mathrm{X} d x}{\mathrm{~A}^{\alpha} \sqrt{\mathbf{R}}}+\int \frac{\mathrm{D}_{x}(\mathrm{Y} \sqrt{\mathbf{R}})}{x \mathrm{~A}^{\alpha}} d x-\frac{\mathbf{Y} \sqrt{\mathbf{R}}}{\alpha \mathbf{A}^{\alpha}},
$$

à laquelle je donnerai cette autre forme

$$
\int \frac{\mathrm{G} d x}{\mathrm{~A}^{\alpha+1} \sqrt{\mathbf{R}}} d x=\int \frac{\mathrm{G}_{1} d x}{\mathrm{~A}^{\alpha} \sqrt{\mathbf{R}}}-\frac{\mathrm{Y} \sqrt{\mathbf{R}}}{\alpha \mathbf{A}^{\alpha}},
$$

en posant, pour abréger,

$$
G_{1}=X+\frac{I}{2 \alpha} Y^{\prime}+\frac{1}{\alpha} Y^{\prime} R .
$$

Un voit que, $G_{1}$ étant un polynôme entier oomme $G$, cette égalité donne une formule de réduction dont l'application répétée conduit à l'expression générale

$$
\int \frac{\mathrm{G} d x}{\mathrm{~A}^{\alpha+1} \sqrt{\mathrm{R}}}=\int \frac{\Pi d x}{\mathrm{~A} \sqrt{\mathrm{R}}}+\frac{\Phi \sqrt{\mathrm{R}}}{\mathrm{A}^{\alpha}},
$$

où II et $\Phi$ représentent des fonctions entières de la variable.

Considérons en second lieu l'intégrale $\int \frac{M d x}{S^{\sigma} \sqrt{R}}$, où $S$ est supposé un diviseur de $\mathrm{R}$, de sorte qu'on peut poser

$$
\mathbf{R}=\mathrm{SU} \text {. }
$$

Cela étant, je détermine deux polynômes entiers $\mathbf{X}$ et $\mathbf{Y}$, par la condition

$$
\mathbf{M}=\mathbf{S X}+\mathbf{S}^{\prime} \mathbf{U Y},
$$

à laquelle on peut ainsi satisfaire, puisque $\mathrm{S}$ et $\mathrm{S}^{\prime} \mathrm{U}$ sont premiers entre eux. Nous obtiendrons par là

$$
\int \frac{\mathrm{M} d x}{\mathrm{~S}^{\tau} \sqrt{\mathrm{R}}}=\int \frac{\mathrm{X} d x}{\mathrm{~S}^{\tau} 1 \sqrt{\mathrm{R}}}+\int \frac{\mathrm{S}^{\prime} \mathrm{UY}}{\mathrm{S}^{\tau} \sqrt{\mathrm{R}}} d x .
$$


puis, en remarquant qu'on peut écrire successivement

$$
\begin{aligned}
\int \frac{\mathrm{S}^{\prime} \mathrm{UY}}{\mathrm{S}^{\sigma} \sqrt{\mathrm{R}}} d x & =\int \frac{\mathrm{S}^{\prime} \mathrm{Y} \sqrt{\mathrm{T}}}{\mathrm{S}^{\sigma+}{ }_{2}^{1}} d x \\
& =-\frac{1}{\sigma-\frac{1}{2}} \int \mathrm{D}_{x}\left(\frac{\mathrm{I}}{\mathrm{S}^{\sigma-\frac{1}{2}}}\right) \mathrm{Y} \sqrt{\mathrm{U}} d x \\
& =-\frac{\mathrm{Y} \sqrt{\mathrm{U}}}{\left(\sigma-\frac{1}{2}\right) \mathrm{S}^{\sigma-\frac{1}{2}}}+\int \frac{\mathrm{D}_{x}(\mathrm{Y} \sqrt{\mathrm{U}})}{\left(\sigma-\frac{1}{2}\right) \mathrm{S}^{\sigma-\frac{1}{2}}} d x,
\end{aligned}
$$

cette égalité prend la forme

$$
\int \frac{\mathrm{M} d x}{\mathrm{~S}^{\sigma} \sqrt{\mathrm{R}}}=\int \frac{\mathrm{X} d x}{\mathrm{~S}^{\sigma-1} \sqrt{\mathrm{R}}}+\int \frac{\mathrm{D}_{x}(\mathrm{Y} \sqrt{\mathrm{U}})}{\left(\sigma-\frac{1}{2}\right) \mathrm{S}^{\sigma-\frac{1}{2}}} d x-\frac{\mathrm{Y} \sqrt{\mathrm{U}}}{\left(\sigma-\frac{1}{2}\right) \mathrm{S}^{\sigma-\frac{1}{2}}} \cdot
$$

Posons, pour abréger,

$$
\mathbf{M}_{1}=\mathbf{X}+\frac{\mathrm{I}}{2 \sigma-1} \mathbf{Y U}^{\prime}+\frac{\mathrm{I}}{\mathrm{I} \sigma-\frac{1}{2}} \mathbf{Y}^{\prime} \mathrm{U}
$$

nous en concluons facilement

$$
\int \frac{\mathrm{M} d x}{\mathrm{~S}^{\sigma} \sqrt{\mathrm{R}}}=\int \frac{\mathbf{M}_{1} d x}{\mathrm{~S}^{\sigma-1} \sqrt{\mathrm{R}}}-\frac{\mathrm{Y} \sqrt{\mathbf{R}}}{\left(\sigma-\frac{1}{2}\right) \mathrm{S}^{\sigma}} \cdot
$$

C'est donc encore une formule de réduction, et dont l'application peut se continuer sans qu'on soit, comme précédemment, arrêté par la présence d'un logarithme. De proche en proche, nous en concluons l'expression générale

$$
\int \frac{\mathrm{M} d x}{\mathrm{~S}^{\sigma} \sqrt{\mathrm{R}}}=\int \frac{\Theta d x}{\sqrt{\mathbf{R}}}+\frac{\mathrm{H} \sqrt{\mathbf{R}}}{\mathrm{S}^{\sigma}},
$$

dans laquelle $\Theta$ et $H$ représentent des polynômes entiers.

Revenons maintenant à l'intégrale hyperelliptique, qui a été représentée par la formule

$$
\int \frac{\mathrm{P} d x}{\mathrm{Q} \sqrt{\mathrm{R}}}=\int \frac{\mathrm{G} d x}{\mathrm{~A}^{2+1} \sqrt{\mathrm{R}}}+\int \frac{\mathrm{H} d x}{\mathrm{~B}^{\beta+1} \sqrt{\mathrm{R}}}+\cdots+\int \frac{\mathrm{M} d x}{\mathrm{~S}^{\sigma} \sqrt{\mathrm{R}}}+\cdots
$$

Les résultats précédemment obtenus donnent la conclusion suivante :

Soit $K=A B C$... le produit des facteurs simples de $Q$, qui 
n'appartiennent pas à $R$; si l'on pose $Q=K L$, on aura

$$
\int \frac{\mathrm{P} d x}{\mathrm{Q} \sqrt{\mathrm{R}}}=\int \frac{\mathrm{F} d x}{\mathrm{~K} \sqrt{\mathrm{R}}}+\frac{\mathrm{G} \sqrt{\mathrm{R}}}{\mathbf{L}},
$$

en désignant par $\mathrm{F}$ et $\mathrm{G}$ des polynômes entiers en $x$.

L'intégrale à laquelle se trouve ainsi ramenée la proposée conduit immédiatement, si l'on décompose la fonction rationnelle $\frac{F}{\mathbf{K}}$ en fractions simples, aux fonctions que l'on nomme de troisième espèce. Mais la partie entière de $\frac{F}{\mathbf{K}}$, que je désignerai par $\mathrm{E}$, nous amène à traiter un dernier point, ayant pour objet la réduction de l'intégrale $\int \frac{E d x}{\sqrt{\mathbf{R}}}$. Dans ce but, j'emploirai le développement en série, suivant les puissances descendantes de $x$, de l'expression suivante :

$$
\frac{1}{\sqrt{\mathrm{R}}} \int \frac{\mathrm{E} d \dot{x}}{\sqrt{\mathrm{R}}}
$$

qu'on obtient facilement, comme on va voir.

Désignons par $r$ le degré de $\mathrm{R}$, et supposons le module de la variable supérieur au module maximum des racines de l'équation $\mathrm{R}=\mathrm{o}$; on aura d'abord

$$
\frac{\mathrm{I}}{\sqrt{\mathrm{R}}}=x^{-\frac{r}{2}} \cdot\left(\alpha+\frac{\alpha_{1}}{x}+\frac{\alpha_{2}}{x^{2}}+\cdots\right) \cdot
$$

Multiplions ensuite par le polynôme $\mathrm{E}$, dont je représente le degré par $e$, il viendra ainsi

$$
\frac{\mathrm{E}}{\sqrt{\mathrm{R}}}=x^{-\frac{r}{2}}\left(\beta x^{e}+\beta_{1} x^{e-1}+\ldots+\frac{\gamma}{x}+\cdots\right),
$$

et, par conséquent,

$$
\int \frac{\mathrm{E} d x}{\sqrt{\mathrm{R}}}=x^{-\frac{r}{2}}\left(\frac{\beta x^{e+1}}{e-\frac{r}{2}+1}+\frac{\beta_{1} x^{e}}{e-\frac{r}{2}}+\cdots\right)
$$

Nous concluons de là, en multipliant de nouveau par $\frac{\mathrm{I}}{\sqrt{\mathrm{R}}}$, qu'on peut écrire

$$
\frac{\mathrm{I}}{\sqrt{\overline{\mathrm{R}}}} \int \frac{\mathrm{E} d x}{\sqrt{\overline{\mathrm{R}}}}=\mathrm{M}+\mathrm{S}
$$


$\mathrm{M}$ étant un polynôme en $x$ de degré $e-r+1, \mathrm{~S}$ une série $\frac{\mathrm{A}}{x}+\frac{A_{1}}{x^{2}}+\cdots$, ̀̀ laquelle s'ajoute, s'il y a lieu, la quantité $\frac{\log x}{\sqrt{\bar{R}}}$, multipliée par une constante. C'est le polynôme M qui conduit à la réduction cherchée. Ayant en effet

je considère l'égalité

$$
\mathrm{D}_{x}(\mathrm{M} \sqrt{\mathrm{R}})=\frac{\frac{1}{2} \mathrm{MR}^{\prime}+\mathrm{M}^{\prime} \mathrm{R}}{\sqrt{\overline{\mathrm{R}}}},
$$

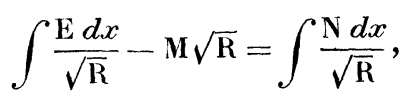

où j'écris, pour abréger,

$$
\mathbf{N}=\mathbf{E}-\frac{1}{2} \mathbf{M R}^{\prime}-\mathbf{M}^{\prime} \mathbf{R}
$$

et je remarque que le degré de $\mathrm{N}$ résulte immédiatement de la relation

$$
\mathrm{S} \sqrt{\mathrm{R}}=\int \frac{\mathrm{V} d x}{\sqrt{\overline{\mathrm{R}}}}
$$

Si on le désigne par $n$, et qu'on développe suivant les puissances descendantes de la variable, on trouvera en effet, en égalant les exposants les plus élevés dans les deux membres, la condition

$$
\frac{r}{2}-\mathrm{r}=n-\frac{r}{2}+1
$$

On a donc $n=r-2$, et l'égalité

$$
\int \frac{\mathrm{E} d x}{\sqrt{\mathrm{R}}}=\int \frac{\mathrm{N} d x}{\sqrt{\mathrm{R}}}+\mathrm{M} \sqrt{\mathbf{R}}
$$

donne, comme nous l'avons dit, la réduction de l'intégrale proposée. Je terminerai en remarquant que l'équation générale précédemment obtenue, à savoir

$$
\int \frac{\mathrm{P} d x}{\mathrm{Q} \sqrt{\mathrm{R}}}=\int \frac{\mathrm{F} d x}{\mathrm{~K} \sqrt{\mathrm{R}}}+\frac{\mathrm{G} \sqrt{\mathrm{R}}}{\mathrm{L}},
$$

ne détermine pas complètement les polynômes F et G. Sans altérer la forme du second membre, on peut, en effet, lui ajouter la quantité identiquement nulle

$$
\int \frac{\frac{1}{2} H R^{\prime}+H^{\prime} \mathbf{R}}{\sqrt{\mathbf{R}}} d x-\mathbf{H} \sqrt{\mathbf{R}}
$$


où $\mathrm{H}$ est un polynôme arbitraire. Mais nous voyons par là qu'on peut toujours, en disposant de ce polynôme, supposer le degré de $G$ moindre que celui de $L$.

Pour plus de clarté, désignons par $f, g, k, l$ les degrés de $\mathbf{F}$, $\mathrm{G}, \mathrm{K}, \mathrm{L}$; on aura ainsi $g=l-\mathrm{r}$, et notre égalité montre, en développant suivant les puissances descendantes de $x$ le terme algébrique et les deux intégrales, qu'il faut prendre $f=r+k-2$, si l'on suppose, comme on peut le faire, le polynôme $\mathbf{P}$ de degré moindre que $Q$. Cela étant, il est facile de voir que $G$ et $F$ se trouvent alors déterminés. Prenons en effet la dérivée de l'équation, et remarquons pour cela que le quotient $\frac{L^{\prime}}{\mathrm{L}}$ est de la forme $\frac{\mathrm{J}}{\mathrm{KR}}$, en désignant par $\mathrm{J}$ un polynòme de degré $r+k-\mathrm{I}$. Un calcul facile nous donne

$$
P=\mathbf{F}+\mathrm{G}\left(\frac{1}{2} \mathrm{KR}^{\prime}-\mathrm{J}\right)+\mathrm{G}^{\prime} \mathrm{KR}
$$

or, le degré par rapport à $x$ de l'identité à laquelle il faut ainsi satisfaire étant $r+k+l-2$, on obtient $r+k+l-\mathrm{I}$ équations qui déterminent, par conséquent, les coefficients de $\mathrm{G}$, au nombre de $l$, et ceux de $\mathrm{F}$, au nombre de $r+k-1$. De là résulte un second procédé que je me contenterai d'avoir indiqué en quelques mots, pour parvenir à la formule générale de réduction des intégrales hyperelliptiques. 